\title{
5 Grundlagen und Formen der Sprachbetrachtung
}

Im Zusammenhang mit der sozialen und individuellen Dimension des Schreibens (vgl. Kap. 3) wurde die Bedeutung von Sprache als Wissenssystem angesprochen und in Bezug auf sprachliche Normen diskutiert. Sprachproduktion geht immer mit (implizitem oder explizitem) Sprachwissen in Form von sprachlichen Normen mit unterschiedlichem Abstraktheitsgrad einher. Das folgende Kapitel knüpft an diesen Aspekt an und geht einen Schritt weiter in der Modellierung dieses sprachlichen Wissens. Es thematisiert dabei grundlegende Kategorien aus der Sprachwissenschaft und geht weiter auf unterschiedliche Konzeptualisierungen von Sprachwissensvoraussetzungen ein. Gleichzeitig zeigt es die Rolle metasprachlicher Kompetenzen für die Schriftsprachaneignung auf. Die Auswahl der im Folgenden diskutierten Konzepte orientiert sich stark an deren Nutzen für einen empirischen Zugang zur metasprachlichen Kompetenz.

\subsection{Zweidimensionalität und Doppelfunktionalität der Sprache}

„Ohne die Sprache würde alles ins Chaos stürzen, weil man nichts versteht. Nicht weiss, was man tun und lassen soll. Daher ist die Sprache unerklärbar.“ ${ }^{20}$ Diese schriftliche Äusserung stammt von einem Jungen einer 6. Primarschulklasse. Er beschreibt Sprache darin als etwas Unerklärbares und ortet die Gründe für diese Unerklärbarkeit in der absoluten Unverzichtbarkeit der Sprache als Mittel der Kommunikation und der Handlungsanleitung. Was genau er selber mit dieser Attribuierung meint, bleibt eine Frage der Interpretation und geht nicht deutlicher aus seinen Ausführungen hervor. Sie trifft die Rolle, die der Sprache - präziser: dem Sprechen- und Schreiben-Können - in der Gesellschaft zukommt, aber mit beachtenswerter Präzision. Der Kommentar kann zudem gelesen werden als Verweis auf eine der Sprache inhärente Qualität, die linguistisch mit den Fachtermini der Doppelfunktionalität und der Autoreferen-

20 Das Zitat von Schüler 6mba12 ist einem Fragebogen entnommen, der in der vorliegenden Studie für die Erhebung sozialer Daten eingesetzt wurde. Die entsprechende Aufgabe - die Kinder waren angehalten, einem ausserirdischen Wesen die menschliche Sprache zu beschreiben und zu erklären - diente den Kindern als Schreibanlass, nachdem sie den Bogen ausgefüllt hatten, eine Auswertung der Antworten war daher nicht vorgesehen.

Ә Open Access. (C) 2018 Pascale Schaller, publiziert von De Gruyter. (c) BY-NC-ND Dieses Werk ist lizenziert unter der Creative Commons Attribution-NonCommercial-NoDerivatives 4.0 Lizenz. https://doi.org/10.1515/9783110555165-005 


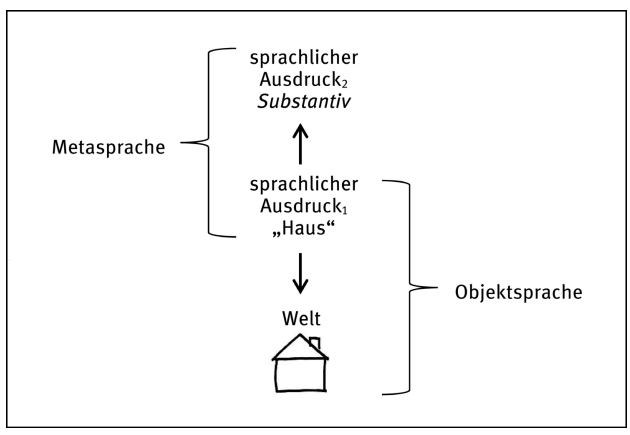

Abb. 5.1: Doppelfunktionalität von Sprache (übernommen aus Bredel 2007: 26).

tialität gefasst wird: Das Mittel, mit dem metasprachlich über etwas kommuniziert wird, ist das gleiche wie der ,Gegenstand', über den gesprochen wird. Die Sprache hat damit eine doppelte Funktion.

Die menschliche Sprache zeichnet sich durch ihre Zweidimensionalität aus: Zeichenbedeutungen und Verknüpfungsregeln weisen jeweils eigene Gesetzmässigkeiten auf. Sie verändern sich über die Zeit hinweg und sind damit dem Sprachwandel unterworfen. Gleichzeitig enthalten sie ein Potenzial zur selbstreflexiven Bezugnahme und zur Sprachbetrachtung. Letztere umfasst einerseits Tätigkeiten des expliziten Kommentierens von Sprachlichem, andererseits aber auch implizite Tätigkeiten wie die Durchführung sprachlicher Operationen, wie sie beispielsweise beim Abwägen verschiedener Formulierungen beim Schreiben zum Ausdruck kommen (Bredel 2007: 23). Der tatsächliche Sprachgebrauch und das konkrete sprachliche Handeln werden also von Aktivitäten der Sprachbetrachtung und Sprachreflexion begleitet. Die terminologische Fassung der beiden Dimensionen, des sprachlichen Handelns und der metasprachlichen Reflexion, führte in der Forschung $\mathrm{zu}$ verschiedenen gebräuchlichen Begriffsdichotomien, die insgesamt ein unübersichtliches Bild dessen ergeben, was unter Sprachhandeln und Sprachreflexion verstanden wird. In der Sprachwissenschaft hat sich - neben anderen Bezeichnungen für diese Dimensionen - die Differenzierung zwischen Objektsprache und Metasprache etabliert. Bußmann \& Gerstner-Link (2002: 433) definieren Metasprache als „Sprache zweiter Stufe (auch: Beschreibungssprache), mittels der die natürliche Sprache (auch: Objektsprache) beschrieben wird“. Die Abgrenzung von Objekt- und Metasprache sei eine beim Sprechen über Sprache notwendige Unterscheidung verschiedener Aussagestufen (Bußmann \& Gerstner-Link 2002: 480).

Diese Unterscheidung zwischen Meta- und Objektsprache ist nicht trennscharf möglich: Das begriffliche Gegensatzpaar umfasst in der Realität ein Kon- 
tinuum, an dessen Enden sich wohl eindeutige Merkmale von Objekt- und Metasprache ansiedeln lassen, das sich aber insbesondere durch einen fehlenden Substanzunterschied zwischen den beiden Polen auszeichnet, wie Abbildung 5.1 visualisiert: Das Lexem Haus wird in objektsprachlichen Äusserungen (Wir sind in ein Haus gezogen.) und in metasprachlichen Kommentaren (Das Substantiv Haus ${ }^{21}$ schreibt man aber gross.) gleichermassen verwendet, und zwar auch im alltäglichen Sprachgebrauch. Laut Andresen \& Funke (2006: 438) sind gerade in der Alltagskommunikation Meta- und Objektsprache miteinander verflochten. Das gilt im Besonderen für die psycholinguistische Definition des Begriffs Metasprache, die breiter ist, als die linguistische Abgrenzung, wie Gombert (1990: 15) präzisiert:

\begin{abstract}
Linguistiquement parlant, est métalinguistique tout ce qui a trait à la métalangue. Autrement dit, le linguiste dépistera « le métalinguistique » en identifiant dans des productions verbales des marques linguistiques traduisant des processus d'autoréférenciation (utilisation du langage pour référer à lui-même), le psychologue quant à lui cherchera dans le comportement (verbal ou non) du sujet des éléments qui lui permettront d'inférer des processus cognitifs de gestion consciente (de réflexion sur, ou de contrôle délibéré), soit des objets langagiers en tant que tels, soit de leur utilisation. (Gombert 1990: 15)
\end{abstract}

Sprache hat also, wie das Beispiel mit dem Substantiv Haus zeigt, die doppelte Funktion der Objektsprache und der Metasprache. Metasprachliche Äusserungen zeigen zudem ihre Autoreferentialität auf: Sprache wird verwendet, um über Sprache zu sprechen. Die Autoreferentialität und die Doppelfunktionalität der Sprache bilden die Grundvoraussetzung und das Mittel für die metasprachliche Verständigung. Darüber hinaus ist aber für Untersuchungen der (meta)sprachlichen Entwicklung wichtig, wie sich das Zusammenspiel von Sprachhandeln und Sprachwissen gestaltet (vgl. Kap. 3.2.3). Metasprache ist die Voraussetzung dafür, über Sprache zu reflektieren und zu sprechen, und damit die Basis von Grammatik- und Normkonzeptionen. Braucht es sie aber auch, um gut zu schreiben und zu sprechen? Wann geht die Metasprache der Objektsprache voraus und wann ist das umgekehrt? Im Hinblick auf Erwerbsfragen betonen Andresen \& Funke (2006) das Ineinanderspielen von Objektund Metasprache und warnen vor der Annahme einer Erwerbsreihenfolge in der Aneignung dieser beiden Arten des Sprachgebrauchs und des Sprachwissens. Gleichzeitig problematisieren sie Begriffsdichotomien, die die Möglichkeit einer Abgrenzung zwischen zwei Bereichen suggeriere:

21 Formale Auszeichnungen dieser Art, wie sie in schriftlichen, speziell in wissenschaftlichen, Texten gebräuchlich sind, markieren die metasprachliche Verwendung von Termini. In der Mündlichkeit hingegen wird ein Substanzunterschied nicht markiert. 
Theoretische Überlegungen zur Entwicklung von sprachlichem Wissen und sprachlicher Bewusstheit sind in den letzten Jahrzehnten durch Begriffsdichotomien wie implizites und explizites, unbewusstes und bewusstes, prozedurales und deklaratives Wissen beherrscht worden [...]. Wenn man sich auf solche Unterscheidungen verschiedener Kategorien von Wissen konzentriert, so führt das leicht dazu, Sprachproduktion und -perzeption zugrunde liegendes Wissen einerseits und metasprachliches Wissen andererseits statisch einander gegenüberzustellen und dabei zu vernachlässigen, dass in der sprachlichen Handlungspraxis Sprachproduktion/-rezeption und Thematisierungen von Sprache gemeinsam auftreten. Somit besteht die Gefahr, die Entstehung solcher verschiedenen Kategorien sprachlichen Wissens als ein striktes Nacheinander zu konzipieren, was insbesondere für theoretische Ansätze gilt, die die Bedeutung des Schriftspracherwerbs für die Entstehung von Sprachbewusstheit ${ }^{22}$ betonen[.] (Andresen \& Funke 2006: 439)

Die Autoren weisen damit darauf hin, dass die Aneignung einer Metasprache Teil des sprachlichen Erwerbsprozesses ist. Zwischen Sprachhandeln und Sprachwissen besteht keine unidirektionale Verbindung in der Art, dass Individuen beim Produzieren sprachlicher Äusserungen auf vorhandenes Wissen zurückgreifen; Sprachwissen also dementsprechend erworben werden muss, um angemessene sprachliche Äusserungen zu realisieren. Gebrauchsbasierte Ansätze betonen aus diesem Grund die Rückwirkung des Sprachhandelns auf das Sprachwissen. Sie modellieren metasprachliches Wissen nicht nur als Ressource, sondern gleichfalls als Ergebnis des Sprachgebrauchs, wie in Kapitel 6 ausführlich gezeigt wird.

\subsection{Bedeutung der Metasprache für die Sprachaneignung}

Die Erforschung kindlicher Metasprache etablierte sich in den 60er und 70er Jahren des 20. Jahrhunderts (Stude: 64). Neben Notizen zur metasprachlichen Entwicklung von Kindern in einzelnen Tagebuchaufzeichnungen (vgl. für einen Überblick Wehr 2001) widmen sich vor dieser Zeit einzig die Arbeiten des Psychologen Vygotskijs aus den 1930er Jahren eingehend der Bewusstwerdung von Sprache. $\mathrm{Zu}$ einer würdigen Rezeption seines sozial-konstruktivistischen Ansatzes auch von westlich orientierten Wissenschaftlern kam es erst 1962 durch die Übersetzung seines Werks Thought and Language. Seine Konzeption von der frühen kognitiven Entwicklung und der zentralen Bedeutung der sozialen Umwelt hat bis heute enormen Einfluss auf entwicklungspsychologische, sprachdidaktische und erziehungswissenschaftliche Theorienbildungen und Konzepte (Stude 2012: 65).

22 Vgl. zur terminologischen Differenzierung zwischen Metasprache und Sprachbewusstheit/ Sprachbewusstsein Kapitel 4.3. 
Wissen über Sprache und Grammatikalitätsurteile bildeten einen zentralen Untersuchungsgegenstand im Rahmen des generativen Paradigmas. Sie wurden als Ausdruck der dem Sprachgebrauch zugrundeliegenden Kompetenz gewertet. Kritiker Chomskys hingegen stellen einerseits dessen Kompetenzbegriff und andererseits die Annahme in Frage, dass Grammatikalitätsurteile valide Indikatoren für metasprachliche Kompetenzen darstellen. Psycholinguistische Untersuchungen kommen denn auch zum Schluss, dass Urteile über die Grammatikalität von Sätzen auf sprachlicher Performanz beruhen und durch Performanzfaktoren ebenso beeinflusst werden können wie andere sprachliche Aktivitäten (Bußmann \& Gerstner-Link 2002: 615). Während sich die generative Transformationsgrammatik für die sprachlichen Intuitionen als Hinweise auf eine dahinterliegende Kompetenz interessierte (Bußmann \& Gerstner-Link 2002: 615), beschäftigten sich andere Forschungsrichtungen mit metasprachlichen Kompetenzen denn auch aus angewandter, didaktischer und pädagogischer Perspektive: So wurde die aus Großbritannien kommende language awarenessKonzeption aus dem Anliegen entwickelt, „eine Brücke zwischen dem muttersprachlichen und fremdsprachlichen Unterricht $\mathrm{zu}$ schlagen. Sie ist im Sinne eines Wissens über Sprache stärker ganzheitlich, soziolinguistisch und auch politisch-gesellschaftlich orientiert“ (Eichler \& Nold 2007: 65). Unter Fokussierung auf die phonologische Bewusstheit, einem Konzept, das in den 1980er Jahren entwickelt wurde, um frühe Lese- und Schreibfertigkeiten zu fördern (Schneider et al. 2013), wurde vor allem untersucht, ob die Fähigkeit zur Segmentierung gesprochener Sprache in ihre Laute eine Voraussetzung für den Erwerb alphabetischer Schriften ist (Rickheit, Sichelschmidt \& Strohner 2002: 136-137; Roos \& Schöler 2009: 11-13; Wehr 2001: 68 und S. 107; Hartmann 2002; Weingarten 2001: 212-213; Gombert 1990). Korrelations- und Trainingsstudien deuten insgesamt auf einen engen Zusammenhang zwischen dem Schriftspracherwerb und phonologischer Bewusstheit hin: Sie identifizieren den genannten Teilbereich sprachlicher Bewusstheit als Prädiktor für Leistungen im Lesen und Schreiben (Stude 2012: 67). Allerdings ist einzuräumen, so Stude (2012: 68),

dass die phonologische Bewusstheit zwar den am gründlichsten untersuchten Teilbereich innerhalb des Forschungszweigs darstellt, jedoch kaum bis gar nicht an ein explizites Thematisieren sprachlicher Einheiten gekoppelt sind. D.h. es ist berechtigt zu fragen, inwiefern hier überhaupt von einem Teilbereich metasprachlicher Fähigkeiten gesprochen werden sollte und worin die Gründe liegen, dass sich der Forschungszweig so stark auf diesen Teilaspekt sprachlicher Bewusstheit verengt hat.

Forschungen zur phonologischen Bewusstheit finden insbesondere in der sprachheilpädagogischen Arbeit Anwendung. In der Logopädie spielen die Psychometrie und die Entwicklung möglichst präziser Verfahren zur Sprach- 
standsbestimmung und zur Diagnose pathologischer Erwerbsverläufe eine herausragende Rolle, während moderne Didaktiken den reflexiven Zugang zur Sprache fordern und fördern. ${ }^{23}$ Sie sind dabei nicht auf Sprachstandsmessungen ausgerichtet, sondern zielen eher im Gegenteil darauf ab, die Kinder ausgehend von ihrem je individuellen Entwicklungsstand $\mathrm{zu}$ fördern, indem sie ihnen einen reflexiven Zugang zu Sprache und zur eigenen Sprachkompetenz ermöglichen. Die unterschiedlichen Modellierungen des Forschungsgegenstandes finden ihren Ausdruck wie oben angesprochen in verschiedenen Begrifflichkeiten, insbesondere in Begriffsdichotomien. Empirisch wurden zudem unterschiedliche metasprachliche (Teil-)Kompetenzen untersucht. Diese Einzelkenntnisse stehen hingegen meist unverbunden nebeneinander. Eine kohärente Überblicksdarstellung des Erwerbs metasprachlicher Fähigkeiten fehlt daher nach wie vor. Begründet ist diese Heterogenität des Forschungszweigs neben der uneinheitlichen Bestimmung des Untersuchungsgegenstandes in den unterschiedlichen Sprachkonzeptionen, die den Untersuchungen zugrunde liegen (Stude 2012: 64).

Unbestritten ist in der Forschung, dass metakommunikative Fähigkeiten in der Ontogenese bereits sehr früh eine Rolle spielen. Laut Bruner (1987) ist sie bereits Teil der Erwachsenen-Kind-Interaktion, bevor das Kind sprechen lernt (Bruner 1987; Andresen \& Funke 2006: 438). Darüber, wann erste metasprachliche Handlungen $\mathrm{zu}$ erwarten sind, ist sich die Forschung allerdings uneinig. Heute liegen stark voneinander abweichende und teilweise widersprüchliche Befunde vor zur Frage, zu welchem Zeitpunkt in der Ontogenese der Erwerb metasprachlicher Fähigkeiten anzusetzen ist. Das ist weitgehend auch darauf zurückzuführen, dass die unterschiedlichen Studien von unterschiedlichen Konzeptionen des Bereichs ausgehen. Vermutungen, dass das Gespräch über Sprache bereits im Vorschulalter einen zentralen Bereich der Sprache ausmacht, äusserte Jakobson bereits in den 1950er Jahren. Gestützt auf beobachtete Sprachkommentierungen sowie spielerische Manipulationen verweisen auch Forschungen Ende der 1970er Jahre auf das Vorhandensein metasprachlicher Fähigkeiten ab dem Kleinkind- und Vorschulalter. Ob spielerische Manipulationen als Indikatoren für metasprachliche Kompetenz $\mathrm{zu}$ werten sind, wenn sie Sprache ja nicht explizit zum Gegenstand machen, ist allerdings umstritten.

23 Vgl. etwa die Ausgabe von Grundschule Deutsch (2011) mit dem Titel Nachdenken über Sprache (darin speziell Andresen 2011). Didaktische Konzepte, die der Sprachreflexion gegenüber dem klassischen Grammatikunterricht den Vorzug geben, sowie Überlegungen zur wissenschaftlichen Auseinandersetzung mit dem Gegenstand wurden in den 1990er Jahren u.a. etwa von Werner Ingendahl, Theodor Diegritz, Albert Bremerich-Vos vorgeschlagen (vgl. dazu die Literaturnachweise in Riegler 2006). 


\subsection{Metasprache und Schriftsprachaneignung}

Spätestens mit dem Eintritt in die Schule tritt neben das Bewusstsein, dass über Sprache gesprochen und reflektiert werden kann, ein weiterer Aspekt: Sprache wird explizit zum Gegenstand der (angeleiteten) Reflexion gemacht und ihre medial und schliesslich auch konzeptionell schriftliche Form wird durch gezielte Intervention gesteuert und gefördert. Konfrontiert werden Kinder im Verlauf der Grundschulzeit damit mit einer neuen Art von Sprachbetrachtung und Sprachwissen. Metasprachliches Reflektieren ist zwar auch mit der Sprachverwendung im mündlichen Kontext verbunden und setzt die Schriftlichkeit nicht voraus. Letztere hat allerdings das Potenzial, metasprachliches Denken zu stimulieren (Ehlich 2007: 25). Der Rolle metasprachlicher Aktivitäten und Fähigkeiten für die Aneignung der Schriftsprache wird im Folgenden nachgegangen.

\subsubsection{Metasprache versus Sprachbewusstsein}

So wie Objektsprache und Metasprache sich nicht trennscharf unterscheiden lassen, ermöglichen strukturelle Merkmale metasprachlicher Äusserungen noch keinen direkten Rückschluss auf metasprachliche Reflexion oder Kompetenz. „Wenn eine Äußerung strukturell gesehen - d.h. ihrem Inhalt und ihrer kommunikativen Funktion nach - als metasprachlich eingestuft werden kann, so zeigt ihr Auftreten nicht notwendigerweise an, dass die Kommunikationspartner wirklich Sprachliches als solches zum Gegenstand des Nachdenkens machen.“ (Andresen \& Funke 2006: 439) Umgekehrt gilt ebenfalls, dass metasprachliches Sprechen nicht die Verwendung bestimmter Termini voraussetzt (Camps, Milian \& Ribas 2000: 104; auf diesen Aspekt wird in Kapitel 5.1 unter dem Aspekt der Doppelfunktionalität der Sprache eingegangen). Der kognitive Aspekt metasprachlichen Sprechens muss darum vom strukturellen unterschieden werden, was vor allem im Zusammenhang mit der Schriftsprachaneignung und der damit verbundenen expliziten Auseinandersetzung der Kinder mit Sprache, wichtig ist. Damit kommt ein weiteres Konzept ins Spiel, das unter dem Begriff Sprachbewusstsein ${ }^{24}$ terminologisch vom Begriff Metaspra-

24 Alternativ dazu wird - etwa von Andresen \& Funke (2006) - der Terminus Sprachbewusstheit verwendet. Laut Eichler \& Nold (2007: 147) ist er speziell in der Fremdsprachenforschung gebräuchlich, während in der Soziolinguistik eher von Sprachbewusstsein gesprochen werde. Terminologisch wird in der vorliegenden Studie nicht zwischen den beiden Begriffen unterschieden. Da sich eine Einschränkung auf einen der Termini aufgrund der zitierten Modelle nicht anbietet, werden sie synonym verwendet. 
che abzugrenzen ist: Sprachbewusstsein definieren Bußmann \& Gerstner-Link (2002: 615) als Synonym für language awareness (vgl. Kap. 5.2 und 5.3) oder metasprachliche Fähigkeit als Wissen über Sprache respektive Fähigkeit zu metasprachlichen Urteilen über sprachliche Ausdrücke. Andresen \& Funke (2006: 439) fassen unter Sprachbewusstheit die Bereitschaft und Fähigkeit zusammen, „sich aus der mit dem Sprachgebrauch in der Regel verbundenen inhaltlichen Sichtweise zu lösen und die Aufmerksamkeit auf sprachliche Erscheinungen als solche zu richten." Von metasprachlichem Wissen hingegen kann ihnen zufolge erst gesprochen werden, „wenn sprachliche Kenntnisse in unterschiedlichen Kontexten zugänglich sind“ (Andresen \& Funke 2006: 439). Während sich also der Terminus Metasprache in der genannten Definition auf die Sprache als ,Material' bezieht, meint demgegenüber Sprachbewusstsein eine menschliche Qualität. Die Begriffe zielen damit auf inhaltlich ganz unterschiedliche Konzepte $\mathrm{ab}$.

Als eine der ersten systematischen Darstellungen des Konstrukts Sprachbewusstheit bei jüngeren Kindern ist die Arbeit von Clark (1978) zu nennen, die davon ausgeht, dass die Sprachbewusstheit eine essentielle und integrale Rolle in der Sprachentwicklung spielt und mit dieser zusammen auftritt. Sie nimmt an und diskutiert etwa anhand von spontanen Selbstkorrekturen, dass das Bewusstsein über Sprache mit dem kindlichen Erwerb von Beginn an einhergehe und für diesen eine Voraussetzung sei. Der Ansatz von Clark wurde von Andresen (1985) einer grundlegenden Kritik unterzogen. Kritisiert wurde Clark insbesondere aufgrund der weiten Begriffsauffassung metasprachlichen Handelns, woraus das Zusammenfassen sehr heterogener Phänomene unter einen Begriff resultiert. Als umstritten gilt zudem die Interpretation frühkindlicher Selbstkorrekturen als Anzeichen bewusster metasprachlicher Aktivität:

Die Selbstkorrekturen, über die Clark berichtet, sind nicht mit Äußerungen über Sprache verbunden, weshalb es hier besonders problematisch erscheint, von metasprachlichen Handlungen zu sprechen. Auf der anderen Seite zeigen sie doch Sprachbewußtheit an, nämlich das Erkennen, daß die produzierten Formen Normverstöße aufweisen. (Andresen 1985: 81, Hervorhebung im Original)

Andresen nimmt hier eine Differenzierung zwischen Sprachbewusstheit und metasprachlichen Handlungen vor: Das Unterscheidungsmerkmal macht sie im Grad der Bewusstheit aus, in der Sprachliches zum Gegenstand der Äusserung wird: Sprachbewusstheit liegt vor, wenn Normverstösse bemerkt oder zumindest vermutet werden; ihre Korrektur hat laut Andresen aber noch nicht zwingend den Charakter metasprachlichen Handelns. Andresen geht mit Clark einig in der Vermutung, dass Kinder sich ihrer selbst versichern, indem sie das Sprachsystem durch Spielereien und Variation ausprobieren und auf diesem Wege Regeln erschliessen. Als äusserst problematisch weist sie die Annahme 
zurück, „daß dieses Üben ein Zeichen besonderer Bewußtheit sei“ (Andresen 1985: 92). Die Annahme basiert auf der These, dass Kinder diejenigen Teile der Grammatik spielerisch variieren, an denen sie intensiv und bewusst arbeiten, was weiter bedeuten könnte, dass sie sich in einem bestimmten Erwerbsstadium eines bestimmten Grammatikbereichs oder einer bestimmten Regel bewusst sind, dieses Bewusstsein aber später $\mathrm{zu}$ unbewusstem Wissen wird. „Demnach wäre das unbewußte (intuitive) Sprachwissen, welches nach Vygotskij im Schulunterricht bewußt gemacht wird, in einer früheren Phase des Spracherwerbs bereits einmal bewußt gewesen.“ (Andresen 1985: 92) Da spontaner Spracherwerb nicht durch explizite verbale Anweisungen gesteuert werde und kein abfragbares Wissen über sprachliche Zeichen, Regeln und Verhaltensweisen produziere, sei es hingegen nicht plausibel, „eine bewußte Kontrolle von Sprache im Stadium des Erwerbs mit späterem Absinken auf die Ebene der Unbewußtheit anzunehmen“ (Andresen 1985: 93). Gleichermassen kritisch äussert sich Andresen dazu, dass Clark Selbstkorrekturen als Indiz für Sprachbewusstheit wertet. Sie setzt dem die These entgegen, „daß Selbstkorrekturen zwar bewußt vorgenommen werden können, daß Bewußtheit jedoch nicht die Voraussetzung dafür ist“ (Andresen 1985: 94).

Andresen (1985) leistete mit ihrer unter dem Titel Schriftspracherwerb und die Entstehung von Sprachbewusstheit publizierten Arbeit einen wesentlichen Beitrag zur Entwicklung eines theoretischen Ansatzes, um das Verhältnis der Schriftsprachaneignung und der Entstehung von Sprachbewusstheit zu klären. Sie beschreibt die Sprachentwicklung als Prozess von einer maximalen Verschränkung sprachlicher und nicht-sprachlicher Handlungen hin zur Aneignung (sprachlicher) Mittel, die fortschreitend eine Verselbständigung sprachlicher Handlungen ermöglichen. Zu Beginn ist das Zeigefeld (Bühler 1934) ${ }^{25}$ bestimmend, verbale Äusserungen sind formal wenig differenziert und bedürfen der nonverbalen Stütze. Parasprachliche Mittel wie Gestik und Mimik bilden zusammen mit den sprachlichen eine Einheit. Wenngleich das auch für Gespräche unter Erwachsenen zutrifft, für die nonverbale Handlungen zum Verständnis häufig unverzichtbar sind, haben diese doch - im Gegensatz zu

25 Sprachliche Zeichen erlangen ihre Bedeutung laut Bühler (1934) erst durch das sprachliche Handeln und können jeweils nur situationsgebunden verstanden werden. Ein Beispiel dafür ist etwa die Verwendung der Partikel hier in einer Äusserung: Dies kann nur verstanden werden, wenn aufgrund der kommunikativen Situation klar ist, welcher Ort damit gemeint ist. Die von Bühler in seinem sozialpsychologischen Zeichenmodell unternommene Trennung zwischen Zeichen des Zeige- und Zeichen des Symbolfeldes etablierte in der Sprachwissenschaft einen wichtigen Forschungsbereich, der pragmatische Aspekte von Texten in den Blick nimmt (Heinemann \& Heinemann 2002, S. 33). Gleichzeitig markiert sie in der Ontogenese schriftsprachlicher Handlungsfähigkeiten verschiedene zentrale Aneignungsetappen. 
den Kindern - die Fähigkeit, bei Bedarf Äusserungen zu formulieren, die ohne Situationsbezug verständlich sind (Andresen 1985: 76-77). Das Symbolfeld der Sprache (Bühler 1934) erschliesst sich den Kindern erst im Verlauf der Ontogenese.

Aufgrund der intensiven Beschäftigung mit metasprachlichen Äusserungen im kindlichen Spiel nimmt Andresen (1985) für die Beschreibung des Konstruktes Sprachbewusstheit eine terminologische Differenzierung vor. Sie unterscheidet zwischen den Begriffen Sprachreflexion, aktueller Bewusstwerdung und eigentlicher Bewusstwerdung in folgender Hinsicht: Sprachreflexion definiert Andresen als jede Art von Nachdenken über Sprache und den sprachlichen Ausdruck davon. Aktuelle Bewusstwerdung meint ein Phänomen des aktuellen Redeaktes, der durch das Modell der Sprachfähigkeit abgebildet wird. Einzelne Einheiten verschiedener hierarchisch angeordneter Ebenen können aus dem Redefluss ausgegliedert und als isolierte Bestandteile betrachtet werden. Diese Ausgliederung wird etwa ausgelöst durch unvorhergesehene Ereignisse wie Verständigungsschwierigkeiten oder Normverstösse, „die zur Folge haben, daß die Konzentration von der Intention und dem Handlungsziel, auf die sie normalerweise gerichtet ist, vorübergehend abgezogen wird“ (Andresen 1985: 102). Von eigentlicher Bewusstwerdung ist nach Andresen dann zu sprechen, wenn „die eigene psychische (sprachliche) Tätigkeit zum Erkenntnisobjekt, dadurch verallgemeinert und willkürlicher Steuerung zugänglich wird“ (Andresen 1985: 102). Sie sei immer mit einer bestimmten Explizitheit und mit der Aneignung wissenschaftlicher Begriffe, d.h. mit dem Aufbau eines systematischen Gefüges von Begriffsrelationen, verbunden (Andresen 1985: 102). Der Operation der Ausgliederung kann aufgrund ihres prozesshaften Charakters und den potentiell metasprachlichen Qualitäten eine Brückenfunktion zukommen zwischen der Sprachbeherrschung und Reflexion über den Gegenstand Sprache (Holle 1999: 65).

Die Unterscheidung verschiedener Bewusstheitsgrade durch Andresen verdient insbesondere darum Beachtung, weil die Gegenüberstellung von implizitem und explizitem Wissen ohne feinere Präzisierung zugunsten eines differenzierteren Modells aufgegeben respektive modifiziert wird. Auf diese Differenzierung wird im folgenden Kapitel eingegangen. Eine Schwachstelle ist allerdings die fehlende empirische Überprüfung des Modells. Da die Arbeit Andresens explizit als theoretischer Beitrag konzipiert ist, soll damit nicht die Modellierung kritisiert, sondern auf die offene Frage hingewiesen werden, wie die unterschiedlichen Arten von Sprachbewusstheit und Sprachwissen operationalisierbar sind. Die Frage, die sich an den Vorgang des Ausgliederns stellt, ist die, was genau ausgegliedert wird. Wie sind die Strukturen zu nennen, die betrachtet und durch diesen Betrachtungsprozess aus ihrem Kontext ausgegliedert werden? 


\subsubsection{Einheiten metasprachlicher Reflexion}

Um sprachliche Grössen zu benennen, die von Kindern metasprachlich reflektiert und kommentiert werden, bedienen sich viele Schulgrammatiken beim Inventar linguistischer Kategorien, die - wie etwa für die Wortarten - auf der lateinisch-griechisch basierten Grammatikkonzeption fussen. Es besteht hingegen berechtigter Zweifel daran, ob damit tatsächlich Grössen gefasst werden, die für die metasprachliche Reflexion (und nicht nur für die systematische Sprachbeschreibung) relevant und dem kindlichen Sprachaneignungsprozess angemessen sind. Andresen (1985: 98) weist darauf hin, dass den Sprechern und Hörern die pragmatische und die semantische Dimension der Sprache relativ gut zugänglich seien. Wenn aber

Einheiten tieferliegender Ebenen aus dem Redezusammenhang isoliert werden, dann sind es zunächst solche des Modells der Sprachfähigkeit [...], die nicht identisch sind mit linguistischen Einheiten. Die Fähigkeit, linguistische Elemente identifizieren zu können, setzt einen eigenen Erkenntnisakt voraus, der in besonderen Lernprozessen angeeignet werden muß und den Wygotski als eigentliche Bewußtwerdung bezeichnet [.] (Andresen 1985: 98, Hervorhebung im Original)

Andresen schliesst daraus auf die Notwendigkeit, einen Terminus in die Diskussion einzubringen, die für die Bezeichnung der metasprachlich ausgezeichneten Strukturen geeigneter ist als linguistische Begriffe. Im Anschluss an Leontev schlägt sie vor, für die Kinder metasprachlich relevante Kategorien nicht als Wörter, sondern als Quant-Wörter und nicht als Sätze, sondern als QuantSätze zu definieren. Das Präfix Quant- markiert die Zugehörigkeit der Kategorien zu psycholinguistischen Tätigkeiten, wie Ossner 1989: 32 treffend resümiert: Es handelt sich bei den Quant-Sätzen

nicht um Sätze, sondern um Äußerungen, also um eine intentional und nicht um eine formal zu beschreibende Ausdruckseinheit, unter Quantwörtern sind Ausdruckseinheiten gefaßt, die sich auf Wahrnehmungseinheiten und nicht auf isolierte Denotate beziehen. Ein Quant-Wort ist demnach ein runder Tisch und nicht nur Tisch. Die Silbe kommt als - kleinste - Einheit vor, nicht nur, weil sie sich zu außersprachlichen Tätigkeiten (Stampfen, Klatschen etc.) leicht parallelisieren läßt, sie ist auch Träger der Satzprosodie (Akzent, Rhythmus, Ton, Intonation) und damit des emotionalen Gehalts. [...] Es handelt sich also um pragmatische Einheiten, die keine mit linguistischen Operationen ermittelbare Größe haben. Sofern es zur Ausbildung einer Begrifflichkeit kommt, handelt es sich nie um eine systematische, sie hat lediglich eine vorübergehende, instrumentelle, auf den augenblicklichen Zusammenhang bezogene Funktion. (Ossner 1989: 32, Hervorhebungen PS)

Andresen trennt damit das Modell der Sprachfähigkeit vom Modell der Sprache: Im Modell der Sprachfähigkeit kommen Einheiten wie Silben (als Grund- 
einheit des motorischen Redeprogramms), Quant-Wort und Quant-Satz vor. Diese Quant-Einheiten zeichnen sich durch ihre Nähe zu linguistischen Einheiten aus, weisen aber nicht deren Ausdifferenzierung auf. Das Modell der Sprache wiederum besteht aus linguistischen Einheiten und linguistischen Termini. Beachtenswert am Ansatz Andresens (1985) ist folgendes: Sie lenkt den Fokus auf Entitäten, die nicht aus dem linguistisch beschriebenen Sprachsystem ableitbar sind, sondern erst und gerade dadurch zu einer Einheit werden, dass die Kinder sie als solche wahrnehmen. Dieser Grundgedanke ist eng verknüpft mit dem Blick auf die Sprache als Sprache im Gebrauch und auf die kognitive und psycholinguistische Relevanz bestimmter sprachlich emergenter Strukturen. Quant-Einheiten sind - wenn auch terminologisch noch gebunden an linguistisch definierte Grössen wie Wort, Satz etc. - vergleichbar mit Konstruktionen oder literalen Prozeduren, wie sie in der aktuellen (Schrift-)spracherwerbsforschung diskutiert werden. Auch wenn sie als solche bei Andresen nicht den definitorischen und sprachtheoretischen Rahmen haben, der ihnen in der Konstruktionsgrammatik zukommt, geht Andresen von metasprachlich und für den Sprachproduktionsprozess relevanten Grössen aus, die mehrere sprachstrukturelle und lexikalisch spezifizierte Einheiten umfassen und die gebrauchsbasiert Relevanz erlangen. Die Auszeichnung von Quant-Einheiten als Wahrnehmungseinheiten mit psycholinguistischer Realität verbindet die Ebene des Sprachgebrauchs und der mentalen Repräsentation, wie es das konstruktionsgrammatische Paradigma ausdifferenziert (vgl. Kap. 6).

Andresens Konzeption der sogenannten eigentlichen Sprachbewusstheit qualifiziert sich dadurch, ,daß eine begriffliche Fixierung der ausgegliederten Einheiten stattfindet, die den Basisstandards einer linguistischen oder grammatischen Theorie entspricht“ (Holle 1999: 66). Während nach Andresen Variationen und Kommentierungen sprachlicher Äusserungen, Sprachspielen, Witzen und Rätseln der Status einer eigentlichen Bewusstwerdung zukommt, da durch sie neue Sinnzusammenhänge eröffnet werden, versteht sie die meisten der anderen metasprachlichen Handlungen etwa durch Ausgliederung als Teil der Sprachfähigkeit. Holle (1999: 66) fasst diesen Aspekt treffend wie folgt zusammen:

Andresen differenziert somit zwischen dem Verfügenkönnen über sprachliche Elemente im aktuellen Sprechen und einer damit verbundenen Möglichkeit zur Ausgliederung einzelner sprachlicher Ebenen als Teil des Produktionsprozesses selbst und der Systematisierung der ausgegliederten Elemente durch eine begriffliche Fixierung in einem entsprechenden ,wissenschaftlichen“ Bezugssystem als eine neue Qualität der Sprachreflexion, die an die Vergegenständlichung von Sprache in Schriftsystemen gebunden ist. Insofern wird die ,eigentliche Bewußtwerdung' an den Schriftspracherwerb gekoppelt[.] (Holle 1999: 66) 
In seiner kritischen Auseinandersetzung mit der Arbeit Andresens bezeichnet Holle (1999: 67) deren Bezug auf wissenschaftliche Begrifflichkeiten als problematisch und kritisiert die Annahme, „daß das (derzeitige) linguistische System als eine (wissenschaftliche) Theorie der Sprache statisch sei oder zumindest als ein statisches System zu konzipieren sei“. Ganz im Gegenteil aber bildeten sich Sprach- und Grammatiktheorien aus der wissenschaftlichen Auseinandersetzung heraus und würden immer wieder umgestaltet. Eine bestimmte linguistische Theorie als Massstab zur Bestimmung von ,eigentlicher Sprachbewusstheit‘ zu wählen, führe laut Holle (1999: 68) dazu, dass das Konstatieren von ,Sprachbewusstheit‘ nicht unabhängig von den Bedingungen eines spezifischen linguistischen Theorierahmens vorzunehmen sei. Holles Kritik mag zum einen berechtigt sein: Sie verweist auf die Gefahr der Verabsolutierung sprachtheoretischer Grundlagen, die für die Empirie und die Praxis weitreichende, mithin negative Folgen hat, und ignoriert, dass Sprache usage based und anhand sprachlicher Konstruktionen und nicht über linguistische Kategorien erworben wird. Zum anderen erscheint sie zu absolut: Metasprachliches Bewusstsein ist wie bereits ausgeführt nicht ausschliesslich, aber wesentlich schriftinduziert. Das Schreiben wiederum ist in den schulischen Rahmen eingebunden, der wie jeder gesteuerte Spracherwerb von spezifischen didaktischen und theoretischen Prämissen ausgeht. Die Kinder sind auf jeden Fall mit einem zielsprachlich auf eine bestimmte Weise modellierten Grammatiksystem konfrontiert und eignen sich dessen Termini an. Schriftsprache und metasprachliches Wissen werden zwar zu Teilen eigenaktiv angeeignet, sind aber gleichzeitig auch einem schulischen Lernprozess unterworfen. Didaktische Settings kommen damit nicht umhin, linguistische Termini $\mathrm{zu}$ verwenden, was auch die Wahl eines spezifischen linguistischen Sprachmodells voraussetzt. Das kindliche Sprachbewusstsein als Ausgangspunkt für die Stimulation metasprachlicher Reflexionsprozesse zu wählen, widerspricht daher nicht dem Vorgang, sich auf bestimmte Termini als Grundlage für die Reflexion zu einigen, wie es auch von Ehlich (2016) vorgeschlagen wird.

\subsubsection{Zwischen implizitem und explizitem Sprachwissen}

Andresen \& Funke (2006: 441) nehmen es als Erfahrungstatsache an, „dass mit dem sprachpraktischen Können nicht zwangsläufig eine Zugänglichkeit sprachlicher Kenntnisse gegeben ist, wie sie bei der Bearbeitung metasprachlicher Aufgabenstellungen erforderlich ist.“ Vielmehr sei von Wissenstypen unterschiedlicher Explizitheits- respektive Implizitheitsgrade ${ }^{26}$ auszugehen.

26 Die Gegenüberstellung expliziten und impliziten Wissens geht ursprünglich auf Michael Polanyi (Polanyi 1966) zurück. Die Termini sind in verschiedene Wissenschaftsdomänen und 
Zwischen ihnen muss es zu Transferprozessen kommen (können), da Sprachbewusstsein zu metasprachlichem Wissen werden kann. Nach Karmiloff-Smith (cop. 1992) ist es ein Hauptcharakteristikum von implizitem Wissen, dass es für bereichsübergreifende kognitive Operationen nicht zur Verfügung steht. Implizites Wissen, verstanden als Befähigung zur richtigen Verwendung einer sprachlichen Struktur (nach Karmiloff-Smith (cop. 1992) level-1 representation), entwickelt sich aber weiter, wenn diese Struktur zuverlässig beherrscht wird (behavioral mastery). Das geschieht durch Umstrukturierung ihrer mentalen Darstellung (representational redescription), die zu explizitem Wissen einer ersten Stufe (level-E1 representation) führt. Das konnektionistisch-konstruktivistische Modell von Karmiloff-Smith schliesst die Unterscheidbarkeit verschiedener Grade der Explizitheit von Wissen ein, was eine Gleichsetzung von implizitem mit sprachpraktischem und von explizitem mit metasprachlichem in Frage stellt (Andresen \& Funke 2006: 441, Karmiloff-Smith cop. 1992, 1986). Es geht von einer sogenannten Re-Repräsentation als Modus des Lernens aus (Karmiloff-Smith cop. 1992: 17); das Modell wird von der Autorin selbst daher auch als $R R$ model bezeichnet: ${ }^{27}$

The RR model attempts to account for the way in which children's representations become progressively more manipulable and flexible, for the emergence of conscious access to knowledge, and for children's theory building. It involves a cyclical process by which information already present in the organism's independently functioning, special-purpose representations is made progressively available, via redescriptive process, to other parts of the cognitive system. In other words, representational redescription is a process by which implicit information in the mind subsequently becomes explicit knowledge to the mind, first within a domain and then sometimes across domains. ${ }^{28}$ (Karmiloff-Smith cop. 1992: 17-18, Kursivmarkierungen im Original)

Karmiloff-Smith beschreibt also einen zyklischen Prozess der Sprachwissensaneignung, in dem Wissensbestände zunehmend bewusster zugänglich werden. Das Phasen-Modell erfasst Unterschiede zwischen implizit bestimmten und zunehmend expliziteren Repräsentationen und damit verschiedene Stufen der Bewusstheit:

in die Alltagssprache eingegangen und werden zur Bezeichnung von der Reflexion und Verbalisierung zugänglichen (expliziten) respektive unzugänglichen (impliziten) Wissensbeständen verwendet.

27 Portmann (2005: 7) diskutiert ausführlich die didaktischen Implikationen der Annahmen von Karmiloff-Smith.

28 Karmiloff-Smith (cop. 1992) legt den Fokus ihrer Ausführungen nicht ausschliesslich auf den Erwerb von Sprache, sondern geht im Gegenteil auf ganz verschiedene Domänen ein und thematisiert auch die Übertragbarkeit von erworbenem Wissen auf andere Bereiche. 
Phase 1 is followed by an internally driven phase during which the child no longer focuses on the external data. Rather, system-internal dynamics take over such that the internal representations become the focus of change. In phase 2, the current state of the child's representations of knowledge in a microdomain predominates over information from the incoming data. The temporary disregard for features of the external environment during phase 2 can lead to new errors and inflexibilities. This can, but does not necessarily, give rise to a decrease in successful behavior - a U-shaped developmental curve. [...] Finally, during phase 3, internal representations and external data are reconciled, and a balance is achieved between the quests for internal and external control. In the case of language, for example, a new mapping is made between input and output representations in order to restore correct usage. (Karmiloff-Smith cop. 1992: 19)

Das Modell von Karmiloff-Smith ist in Bezug auf die Sprachaneignung deswegen so aufschlussreich, weil es einen Erklärungsansatz dafür bietet, wie sprachliche Handlungsroutinen, die Individuen vollziehen, ohne über ein explizites Wissen darüber zu verfügen (information in the mind), allmählich zu zugänglichem Wissen (explicit knowledge to the mind) werden. Sie wechseln nicht vom einen Stadium in das andere, sondern durchlaufen (mindestens) zwei Zwischenstufen. In Phase 3 angekommen, zeichnen sie sich schliesslich durch ihre Generalisierbarkeit und Übertragbarkeit auf andere Strukturen aus. Zudem versteht das Modell die Aneignung komplexer sprachlicher Einheiten auf der Basis zugänglicherer Strukturen und dementsprechend als Ausbau bereits vorhandener Konstruktionen. Der Ansatz, der Restrukturierungsprozesse des Sprachwissens zu beschreiben erlaubt, wird dadurch anschlussfähig an das Konzept der Grammatikalisierung, das - ursprünglich als Beschreibung diachroner Sprachwandelprozesse konzipiert - ebenfalls die Herausbildung sprachlicher Strukturen aus bereits bestehenden modelliert (vgl. für die Diskussion in Bezug auf die Schriftsprachaneignung Feilke, Kappest \& Knobloch 2001). Dem $R R$ model und dem Grammatikalisierungskonzept gemeinsam ist die Annahme der anhaltenden Dynamik: Die sprachlichen Grössen sind beim Sprecher und Schreiber dauerhaft Umstrukturierungs- und Grammatikalisierungsprozessen unterworfen. Weiter gehen sie beide von einem Nebeneinander verschieden stark umstrukturierter respektive grammatikalisierter Grössen aus. Komplexere (Wissens-)strukturen müssen einfachere, aus denen sie hervorgegangen sind, nicht ersetzen, sondern ergänzen sie zumindest vorerst noch. Gleiches gilt für weniger und stärker grammatikalisierte Konstruktionen, die diachron parallel in Gebrauch sein können. ${ }^{29}$

29 Auf das Grammatikalisierungsparadigma kann in der vorliegenden Arbeit nicht eingegangen werden. Es sei aber darauf hingewiesen, dass Karmiloff-Smith mit der representational redescription einen Prozess auf der kognitiven Ebene beschreibt, der jener der Reanalyse aus der diachronen Sprachforschung ähnlich ist. In Feilke, Kappest \& Knobloch(2001) werden Mechanismen des Spracherwerbs aus ontogenetischer Perspektive mit Mechanismen des Sprach- 
Warum verdient gerade in Bezug auf die schriftliche Sprache das von Karmiloff-Smith erarbeitete Phasen-Modell besondere Beachtung? Sprachbetrachtung setzt Schriftlichkeit nicht voraus, sie erreicht durch sie allerdings ein neues Potenzial. Der Kontakt mit der Schrift macht aus impliziten Wissensstrukturen explizites Wissen, gleichzeitig kann explizites Wissen verinnerlicht sein oder verinnerlicht werden.

\begin{abstract}
There are many gradations between tacit and explicit knowledge. As well as explicit knowledge which can be both articulated orally and enacted in their writing, writers may have explicit knowledge which they have temporarily forgotten; or explicit knowledge which they can articulate orally but do not transfer into their writing; or tacit knowledge which is not articulated but can be deployed in their writing. Linked to this is the distinction in cognitive psychology between ,declarative knowledge' (knowing that) and ,procedural knowledge' (knowing how); knowledge about grammar is not the same as knowing how to make effective and appropriate grammatical choices. Moreover, the assumption that the continuum moves from tacit knowledge to implicit knowledge is not always true, particularly with learners of English ${ }^{30}$ as an additional language or weaker writers. For these writers, instruction about linguistic features may generate explicit knowledge where there is no corresponding tacit knowledge. (Myhill 2005: 88)
\end{abstract}

Myhill (2005) weist hier auf einen zentralen Aspekt hin, der anknüpft an die Funktion von (insbesondere transitorischen) Normen: Sie könnten dort eine wichtige Rolle spielen, wo implizites Sprachwissen fehlt, insbesondere also bei sprachschwachen Kindern. Sie würden eine Art Steigbügelfunktion übernehmen und die Kinder auf der Ebene der Performanz unterstützen, auch wenn ihnen implizites Wissen noch fehlt. Explizites Wissen über sprachliche Strukturen und über das Schreiben könnte sprachschwachen Kindern helfen, angemessene Sätze und Texte zu produzieren, wenn es ihnen noch an Sprach- und Schreibroutine fehlt. Die Voraussetzung dafür ist ein metasprachlicher Austausch. Diesem Aspekt wird in der vorliegenden Studie empirisch nachgegangen (vgl. dazu die Analysen in Kap. 11.2.4).

Wie sich die Umbauprozesse zwischen den verschiedenen Wissensebenen gestalten, bedarf zu grossen Teilen nach wie vor der empirischen Klärung. Dass sie komplex sind, wird im Folgenden mit Bezug auf den Orthographieerwerb exemplarisch aufgezeigt.

wandels i.S. der Grammatikalisierung sprachlicher Strukturen verglichen. Als Erweiterung dazu für die kognitive Ebene könnte das Konzept von Karmiloff-Smith hinzugezogen werden. Diese Verbindungslinie wird hier nicht vertieft, da sie für den empirischen Teil der Arbeit nicht zielführend ist. Ihr nachzugehen, würde sich in einem anderen Kontext aber durchaus lohnen. 30 Myhill bezieht sich hier auf ein englischsprachiges Korpus, der beschriebene Umstand - so ist plausibel zu vermuten - trifft aber auf L2-Erwerbssituationen und auf schwache Schreiber grundsätzlich zu. 


\subsubsection{Sprachwissen und Orthographieerwerb}

Mit den Ausführungen zu Andresen und Karmiloff-Smith wurden Konzepte vorgestellt, die zwei wesentliche Aspekte für die Modellierung metasprachlicher Kompetenzen betreffen: Die Verbindung zum Schriftspracherwerb und die Frage nach der Zugänglichkeit respektive Unzugänglichkeit metasprachlichen Wissens. Der Bereich der Orthographie stellt innerhalb der Schriftsprachaneignung insofern eine spezielle Domäne dar, als die vorgegebenen Normen kodifiziert und strikter sind als etwas Textsortenkonventionen. Forschungsmethodisch zeigt sich der Sonderstatus der orthographischen Teilkompetenz darin, dass aus Studien zum Erwerb der Textkompetenz orthographische Aspekte oftmals explizit ausgeklammert (und die Kindertexte vor den Analysen sprachformal normiert) werden. Das ist dann methodisch nicht mehr möglich, wenn durch Keystroke Logging erfasste Schreibprozesse im Zusammenhang mit konzeptionell schriftlichen Schreibfähigkeiten fokussiert werden oder wenn es den Kindern beim Beobachten einer Textentstehung selbst überlassen wird, welche sprachlichen Phänomene sie kommentieren wollen, wie es die vorliegende Studie tut. Aus diesem Grund wird diesem sprachlichen Teilbereich an dieser Stelle Rechnung getragen, ohne dabei ausführlich auf die umfangreiche Forschung zum Orthographieerwerb einzugehen. ${ }^{31}$ Es wird daher im Folgenden exemplarisch auf die Studie von Weingarten (2001) eingegangen, und zwar deshalb, weil sie einen empirischen Zugang zur Erhebung metasprachlichen Wissens darstellt und die Ausführungen oben um eine mögliche Operationalisierung von Sprachwissenskategorien ergänzt.

In den Ausführungen zur Schreibentwicklung wurde betont, dass sich die Verarbeitung von sprachlichen Normen und Normierungen durch Kinder im Lernprozess als Teil des Spracherwerbs zwischen sozialen sprachlichen Konventionen und selbstgesteuertem Wissensaufbau bewegt. Gleiches gilt für die um den Terminus von Ferreiro (1999) wieder aufzunehmen - Psychogenese metasprachlicher Kompetenzen: Sie entwickeln sich aus der Spracherfahrung heraus, werden massgeblich aber auch beeinflusst durch bestehende Normen. Die Untersuchung von Weingarten (2001) stellt die Frage ins Zentrum, welche Rolle das Sprachwissen für den Erwerb orthographischer Regeln spielt. Weingarten schlägt eine Einteilung sprachlichen Wissens in vier Stufen vor, wobei diese sowohl primärsprachliches als auch metasprachliches Wissen umfassen (Weingarten 2001):

31 Vgl. dazu etwa Maas (1992); Scheele (2006); Neef (2005); Hensel (2016); Hoffmann-Erz (2015); Karg (2008); Krauss (2014); Nickel (2006); Röber-Siekmeyer (2015); Thomé (1999); Bredel (2011); Röber-Siekmeyer (1999); Reichardt (2015); Kruse \& Reichardt (2016a); Fay (2010); Fay \& Berkling (2013). 
- Als sprachliches Wissen I wird die situativ und intentional angemessene bzw. korrekte Sprachverwendung, also die Realisierung sprachlich korrekter und dem kommunikativen Rahmen angemessener sprachlicher Äusserungen, bezeichnet. ${ }^{32}$ Zum Ausdruck kommt es als sogenanntes Monitoring, wenn Sprecher oder Schreiber die eigenen Äusserungen spontan selber korrigieren.

- Sprachliches Wissen II findet seinen Ausdruck in Urteilen über sprachliche Äusserungen hinsichtlich deren Identität, Grammatikalität oder situativen Angemessenheit sowie in der Anwendung grammatischer Operationen auf sprachliche Äusserungen (segmentieren, umstellen, ersetzen usw.). „Diese Aktivitäten bilden einerseits das Handwerkszeug der linguistischen Arbeit, sie machen aber auch den Grundstock des Grammatikunterrichts aus.“ (Weingarten 2001)

- Als sprachliches Wissen III benennt Weingarten die alltagssprachliche Bezeichnung sprachlicher Einheiten, Funktionen und Operationen. Es unterscheidet sich vom vorangehenden Wissenstyp (sprachliches Wissen II) durch die Verwendung metasprachlicher Äusserungen.

- Die Verwendung fachsprachlicher Bezeichnungen neben alltagssprachlichen wird als sprachliches Wissen IV angesehen.

In der Ryle'schen Unterscheidung zwischen knowing how und knowing that entsprechen die Stufen I und II dem knowing how und die Stufen III und IV dem knowing that. ${ }^{33}$ Die vierte Wissensform nach Weingarten betont den Gebrauch fachsprachlicher Termini, was den Bezug auf ein konkretes Sprachsystem und ein Grammatikmodell impliziert, wie es Holle an Andresens Modellierung kritisiert. In der Untersuchung von Weingarten geht es nun genau um diesen Aspekt: um metasprachliche Kommunikation im schulischen Rahmen und die Frage, welche Rolle schulisch vermittelte Normen beim Verschriften von Wörtern und Sätzen spielen. Weingarten zeigt auf, wie Kinder über Fehlschreibungen in einem Text sprechen und macht an Einzelfällen deutlich, „dass die Zusammenhänge zwischen den verschiedenen Wissensformen äußerst komplex sind und keine einfachen Umsetzungen der höherstufigen Wissensformen

32 In der Tradition der generativen Grammatik wird diese Fähigkeit als Ausdruck sprachlichen Wissens angesehen (Weingarten 2001).

33 Die terminologische Trennung von Ryle hat unlängst in verschiedene Bereiche der Lernforschung Eingang gefunden, bezieht sich ursprünglich aber keineswegs auf Erwerbsmechanismen von Kindern. Es handelt sich dabei vielmehr um eine Auseinandersetzung mit dem Begriff des Intellekts in Bezug auf praktische und theoretische Aktivitäten und um das Verhältnis zwischen Wissen (knowing that) und Können (knowing how) (Ryle 1949). 
stattfinden“ (Weingarten 2001: 220). Schülerinnen und Schülern des 2. bis 4. Schuljahres wurden Texte vorgelegt mit der Bitte, diese auf Fehlschreibungen zu überprüfen. Die Gespräche der Kinder über die aufgefundenen Fehlschreibungen wurden wie die vorgenommenen Korrekturen aufgezeichnet. Die von Weingarten (2001) präsentierten Beispiele beziehen sich ausschliesslich auf die orthographischen Bereiche Phonographie und Gross- und Kleinschreibung. Obwohl den Kindern Texte mit vollständigen Sätzen vorgelegt wurden, beurteilten sie jeweils nur isolierte Wörter. Bezogen auf die Phonographie sowie auf die Gross- und Kleinschreibungen wurden folgende Phänomene beobachtet: Die Kinder nehmen Korrekturen vor und verbalisieren als Begründung falsches sprachliches Wissen IV, das allerdings „so isoliert [erscheint], dass es die richtige Schreibung nicht behindert“ (Weingarten 2001: 221). Ein falsches Verfahren respektive der Bezug auf falsches Sprachwissen führt in diesen Fällen trotzdem $\mathrm{zu}$ einem richtigen Ergebnis respektive vermag dieses nicht $\mathrm{zu}$ verhindern. In anderen Fällen wissen die Kinder die angemessene Korrektur vorzunehmen und können das entsprechende Wort richtig schreiben, kennen aber keine Gründe für die entsprechende Schreibung.

Die verschiedenen Beispiele ermöglichen keine generalisierbaren Resultate, lassen aber Rückschlüsse auf im Unterricht vermittelte Regeln oder Operationen $\mathrm{zu}$, die sich teilweise „in weiten Teilen grundlegend davon [unterscheiden], wie in der neueren Forschung das Schriftsystem und die Orthographie beschrieben werden“ (Weingarten 2001: 210). Die Gesprächsausschnitte weisen darauf hin, dass die Kinder zwar eine beachtliche Anzahl von Regeln und Verfahren kennen und diskutieren und sich damit auf explizites Wissen beziehen, dass dieses Wissen aber für die Entscheidungen im Verschriften folgenlos bleiben. Weingarten zieht denn auch mit Blick auf die von ihm angeführten Ausschnitte aus dem Korpus den Schluss, „dass die Entscheidung über die richtige Schreibung von dem chaotischen und zu einem guten Teil falschen sprachlichen Wissen relativ unabhängig ist“ (Weingarten 2001: 223). Vor dem Hintergrund, dass der Deutschunterricht viel Zeit in die Vermittlung von orthographischem Wissen investiert und dabei auf Regeln und Verfahren zurückgreift, „die aus linguistischer Sicht [häufig] als falsch angesehen werden“ (Weingarten 2001: 210) müssen, sind das beachtenswerte Ergebnisse. Sie lassen zwei Vermutungen zu: (1) Verschiedene Regeln, die die Kinder erwerben, sind aus linguistischer Sicht unzureichend, (2) ob die Kinder über Regelwissen verfügen oder nicht, beeinflusst ihre Rechtschreibkompetenz nicht wesentlich; der Orthographieerwerb stellt sich als zu wesentlichen Teilen eigenaktiv aufgebauter Bereich des Schreibens dar. Zum Nachweis, ob diese Vermutungen tatsächlich zutreffend sind, bräuchte es umfassendere Forschungsergebnisse, als die Studie Weingartens und die deskriptiv-statistischen Analysen sie liefern. Im empi- 
rischen Teil dieser Arbeit wird ein Beitrag $\mathrm{zu}$ dieser Diskussion geleistet (vgl. Kap. 11.4.3). Interessant ist die zweite Vermutung speziell darum, weil in der Spracherwerbsforschung der orthographische Bereich immer wieder gesondert von anderen sprachlichen Aspekten der Schriftsprachkompetenz betrachtet wird. In diesem Zusammenhang sei an das Modell von Berninger \& Winn (2008) (vgl. Abb. 4.1 in Kap. 4.2) erinnert, in dem die Orthographie einen speziellen Teilaspekt der Textgenerierung bildet: Sie macht zusammen mit dem Schreibmedium (Handschrift, Tastatur) und dessen Beherrschung den Teilprozess der Transkription aus, während etwa das Revidieren, also die Arbeit am Textmaterial, den exekutiven Funktionen und damit einem anderen Teilprozess der Textgenerierung zugerechnet wird. Die Orthographie gehört laut Berninger \& Winn (2008) zu den Grundfertigkeiten des Schreibens. Da schliesst sich mit Blick u. a. auf die Studie von Weingarten die Frage an, ob auch das metasprachliche Wissen über die Orthographie einen eigenen Bereich sprachlichen Wissens darstellt. Dafür spricht die besonders enge und verbindliche Kodifizierung orthographischer Normen im Gegensatz zu (weitgehend impliziten) Konventionen und Routinen auf der Textebene. Diese explizite Normierung könnte weiter vermuten lassen, dass der Orthographie besonders auch explizites Normwissen zugrunde liegt. Gerade diese Annahme aber stellt die Studie von Weingarten in Frage. Auch jüngere empirische Forschungsarbeiten zum Orthographieerwerb weisen darauf hin, dass der Zusammenhang zwischen verschiedenen Graden expliziten orthographischen Wissens komplex ist. Hingewiesen sei hier speziell auf die umfangreichen Studien von Fay (2010) und Reichardt (2015), die zwischen verschiedenen Settings der Rechtschreibdiagnostik unterscheiden und empirisch nachweisen, dass die Kompetenzen der untersuchten Kinder im freien Schreiben nicht mit jener bei der Verschriftung von diktierten Einzelwörtern und Sätzen gleichgesetzt werden können. ${ }^{34}$

Mit Bezug auf die oben exemplarisch eingehender präsentierte Studie von Weingarten (2001) sei an dieser Stelle auf einen forschungsmethodischen Aspekt hingewiesen, auf den in Zusammenhang mit der Definition und der Funktion von Normen (vgl. Kap. 3.2.3) bereits aufmerksam gemacht wurde und der in der später folgenden Analyse der metasprachlichen Kommentare der Kinder deutlich wird: Sprachwissen in Form orthographischer Regeln lässt sich verhältnismässig präzise operationalisieren und in Abhängigkeit zur Kompetenz im Sprachhandeln bringen. Für Letzteres gibt es im Bereich der Rechtschreibung jeweils nur eine, im Falle alternativer Schreibungen allerhöchstens zwei

34 Vgl. zur Rechtschreibdiagnostik auch Siekmann (2014b), darin zur Förderung schwacher Kinder speziell Siekmann (2014a). 
korrekte Umsetzungen. Das gilt für viele andere Bereiche der Schreibkompetenz, für die Ausbildung der konzeptionellen Schriftlichkeit, den Erwerb von Prozeduren und Textroutinen usw., nicht. Weiter macht die Untersuchung deutlich, dass Sprachwissen und Reflexionen über Gründe für sprachliches Handeln nicht zwingend und unmittelbar in einem Zusammenhang zum sprachlichen Handeln und zur Sprachkompetenz stehen. Sie illustriert damit, dass auf dem Kontinuum zwischen explizitem und implizitem Wissen einerseits und zwischen Sprachwissen und Sprachhandeln andererseits von komplizierten Umbauprozessen auszugehen ist. Angeführt sei an dieser Stelle zudem der methodische Hinweis, dass das Erhebungssetting, wie es Weingarten vorschlägt und wie es auch in der vorliegenden Studie gewählt wurde, Gespräche von Kindern über das Schreiben und Verschriften aufzeichnen, was genau genommen aber nicht heisst, dass tatsächlich diese Art von Reflexion oder diese Sprachwissensformen während des Schreibens selber entscheidend waren. Das (retrospektive) laute Denken ist aufschlussreich für die Frage, welche Wissensbestände von den Kindern verbalisiert und metasprachlich verhandelt werden, erlaubt aber keinen direkten Rückschluss auf tatsächlich ablaufende Prozesse während des Schreibens. Für die vorliegende Studie ist diesem Umstand im Rahmen der qualitativen Datenanalyse in Kapitel 11.2 Rechnung zu tragen: Die Auswertungen werden der Frage nachgehen, welche Sprachwissenskategorien sprachstarke und sprachschwache Kinder frequent kommentieren und metasprachlich verhandeln. Darauf aufbauend können unter Mitberücksichtigung anderer Daten - etwa der aufgezeichneten Schreibprozesse - Rückschlüsse auf die Relevanz dieser Kompetenz für das Schreiben selbst gewagt werden. Sie nähern sich der Beziehung zwischen metasprachlichem Wissen und Sprachhandeln aber immer nur an und bilden sie keinesfalls direkt ab.

\subsection{Bestimmungsmerkmale und Referenzbereiche metasprachlicher Äusserungen}

Die vorangehenden Ausführungen widmeten sich der terminologisch und inhaltlich herausfordernden Frage, wann eine sprachliche Äusserung als metasprachlich einzustufen ist. Werden diese Äusserungen eingebettet in den Gesprächskontext betrachtet, erweitert sich der Fokus auf den metasprachlichen Diskurs als relevante Einheit, was andere Bestimmungsmerkmale ins Zentrum rückt. Bredel (2007: 23-25) führt als Bestimmungsmerkmale metasprachlicher Diskurse die Distanzierung vom betrachteten Gegenstand, die Deautomatisierung von Handlungsroutinen und die Dekontextualisierung des Phänomens aus seinem ursprünglichen Kontext an. Sprachliche Erscheinungen, so Bredel 
(2007: 23), können einer reflektierenden Auseinandersetzung erst zugänglich werden, wenn eine Distanz des Beobachters zum Beobachteten hergestellt werden kann. Eine Distanznahme geschieht in alltäglichen und schulischen Situationen automatisch oft dann, wenn Handlungsroutinen versagen: Der Tätigkeit, die im Normalfall ohne bewusste Aufmerksamkeitssteuerung durchgeführt wird, kommt dann Beachtung zu. Wenn ein sprachliches Phänomen zum Gegenstand der Aufmerksamkeit werden soll, muss die Automatisierung, die es ermöglicht, dass Sprachproduktion und Rezeption in der Regel ohne spezielle Aufmerksamkeit des Produzenten oder des Rezipienten ablaufen, aufgegeben werden. Erst dadurch können andere kognitive Prozesse aktiviert werden, die Sprachbetrachtungsaktivitäten ermöglichen. Die Deautomatisierung ermöglicht die bewusste Betrachtung einer Handlungsroutine, führt aber notwendigerweise gleichfalls $\mathrm{zu}$ akuten Verstehensverlusten bei der primären Sprachwahrnehmung bzw. -produktion: Der Versuch etwa, sich während des Lesens merken zu wollen, aus wie vielen Wörtern ein Satz besteht, führt automatisch zu Leseverzögerungen.

Mit der Deautomatisierung geht eine Dekontextualisierung einher. Wird die Wortart eines Wortes bestimmt, kommt es zu einer Herauslösung von Wörtern aus ihrem Satzzusammenhang zugunsten der Zusammenführung zu Klassen bestimmter Merkmale. Bredel (2007: 24) bezeichnet die Dekontextualisierung als dritte notwendige Bedingung für Sprachbetrachtung. Sie sei eine typische Vorgehensweise für Betrachtungsvorgänge aller Art: In der Biologie würden Zellen aus Organen isoliert, um sie der Betrachtung zugänglich zu machen, und im Grammatikunterricht Texte nach Substantiven durchsucht. In Bezug auf die Dekontextualisierung bedürfen die für die vorliegende Untersuchung erhobenen Daten (recalls) besondere Aufmerksamkeit: Als Input für metasprachliche Kommentare und Erklärungen wurde den Kindern ihre eigene Textproduktion vorgelegt. Voraussetzung dafür, diese analytisch zu zerlegen und $\mathrm{zu}$ kommentieren, ist - mit dem Terminus Bredels - einerseits die Distanzierung von der beobachteten Schreibhandlung und der dadurch ermöglichte Zugang über eine metareflexive Ebene. Andererseits erfordert das Kommentieren von den Kindern einen Prozess der Dekontextualisierung des kommentierten Bereichs. Beide Vorgänge bilden notwendige Voraussetzungen für die gedankliche Ausgliederung einer Schreibhandlung aus dem Gesamtprozess und ihre Zuführung zu einem metasprachlichen Kommentieren. An dieser Stelle soll darauf aufmerksam gemacht werden, dass es eines zusätzlichen Handlungsschritts bedarf, den Bredel unerwähnt lässt: Eine inhaltlich plausible Begründung und Erklärung einer Beobachtung erfordert nicht nur das Herausheben eines Phänomens aus seinem Verwendungszusammenhang (Dekontextualisierung), sondern ebenfalls den Rückbezug auf diesen Gebrauchskontext. 
Dieser begründende Rückbezug auf den syntaktischen Zusammenhang hängt mit der Einsicht der Funktion einer sprachlichen Grösse zusammen und dürfte darum für die Kinder eine besonders wichtige, wenn auch anspruchsvolle Fertigkeit darstellen. Gerade sprachschwächere Kinder könnten Mühe darin bekunden, auf explizites Sprachwissen zurückzugreifen und dieses auf ein sprachliches Phänomen in seinem Verwendungszusammenhang $\mathrm{zu}$ beziehen. Bredels Termini sollen darum hier um den Begriff der Rekontextualisierung erweitert werden.

Bei den Bestimmungsmerkmalen Distanzierung, Deautomatisierung und Dekontextualisierung handelt es sich um kognitive Fähigkeiten, die den metasprachlichen Diskurs auszeichnen. Folgt man Bredel, müssen diese drei Aspekte erfüllt sein, um einen Diskurs als sprachbetrachtenden Diskurs bezeichnen zu können: Distanz, Deautomatisierung und Heraushebung aus dem Kontext ermöglichen erst das Sprechen über Sprache mit Sprache. Metasprachliche Kompetenzen äussern sich demzufolge darin, inwiefern ein Kind zum sprachlichen Gegenstand in Distanz treten kann, ob es die Sprachproduktion zu deautomatisieren vermag und so der Reflexion zugänglich machen kann und ob es ein bestimmtes Phänomen aus dem Kontext herauszuheben und von diesem unabhängig zu diskutieren vermag. An welchen sprachlichen Merkmalen anschliessend eine Äusserung als metasprachlich zu qualifizieren ist respektive wann eine Äusserung eine metasprachliche Äusserung darstellt, wird in der Forschung - wie es die vorangehenden Ausführungen etwa zu den Quant-Einheiten deutlich gemacht haben - uneinheitlich beantwortet. Sie ist für die vorliegende Untersuchung von untergeordneter Relevanz: Durch das Setting (vgl. für eine Beschreibung des Erhebungssettings Kap. 9.1.6) werden Äusserungen elizitiert, die metasprachlichen Charakter haben, da es sich explizit um Gespräche über sprachliche Aspekte handelt, was gegenüber den Kindern auch explizit als Gesprächsgegenstand genannt wurde. Die Kommentare sind allesamt mindestens insofern metasprachlich, als ihr Input sprachliche Phänomene sind. Es zeigt sich hier vor dem Hintergrund von Bredels Bestimmungsmerkmalen allerdings deutlich, dass gewissen Kindern die Distanznahme zum Text oder die Dekontextualisierung gerade nicht $\mathrm{zu}$ gelingen scheinen und ihre Kommentare wohl sprach- respektive schriftinduziert sind, aber eigentlich nicht Funktion und Struktur metasprachlicher Reflexion ausweisen. Ersichtlich wird anhand der Daten hier allerdings gleichermassen ein Methodenproblem, da sich die Frage aufdrängt, wie die Bredel'schen Bestimmungsmerkmale operationalisierbar sind. Darauf wird im Zusammenhang mit der Datenauswertung am Rande eingegangen. Der Fokus hingegen liegt für die Datenauswertung weniger bei den strukturellen Charakteristika der Kinderäusserung als viel- 
mehr bei der Frage, auf welche Bereiche sich die Äusserungen beziehen und welche sprachlichen Formen, Regeln und Bezeichnungen die Kinder dabei heranziehen.

Stude legt eine Untersuchung vor, die sich der Funktion metasprachlicher Äusserungen in freien Gesprächen von Kindergartenkindern widmet. Sie geht davon aus, dass das Sprechen über Sprache genau wie Sprache selbst in Interaktion erworben wird, und definiert als empirisch zu erreichendes Untersuchungsziel, „die in der Interaktion verankerten und als erwerbsrelevant postulierten Ressourcen im Einzelnen freizulegen“" (Stude 2012: 10). Dafür wird als Ausgangsthese angenommen, „dass Kontexte, in denen auf Sprache Bezug genommen wird, einen ontogenetisch funktionalen Beitrag leisten für den kindlichen Aufbau metasprachlicher Kompetenzen“ (Stude 2012: 19). Dass der Fokus u. a. auf Peer-Interaktionen liegt und aus dem freien Gespräch metasprachliche Sequenzen extrahiert und auf ihre Funktionalität im Hinblick auf Erwerbsressourcen diskutiert werden, unterscheidet die Untersuchung in Bezug auf Methode und Erkenntnisinteresse von anderen Studien, die metasprachliche Kompetenzen stärker anhand vorgefertigter Aufgaben testen. In einer quantifizierenden Beschreibung wird von Stude freigelegt, inwieweit die Kinder in ihrem Kindergartenalltag in Sprachthematisierungen eingebunden sind, und untersucht, „welche Aspekte von Sprache von und gegenüber Kindern thematisiert werden, wer (Kind, Erwachsene) jeweils Initiator solcher Sprachthematisierungen ist, wie ausgebaut metasprachliche Gesprächssequenzen sind und welche Redeanteile dem Kind dabei zukommen“ (Stude 2012: 20, Kursivmarkierung im Original). Als zweiter Analyseschritt werden diese metasprachlichen Aktivitäten im Zusammenhang mit ihrem interaktiven Entstehungsprozess betrachtet, woraus gesprächsanalytisch sogenannte Praktiken metasprachlichen Handelns rekonstruiert werden sollen (Stude 2012: 19-20). Für die vorliegende Studie ist die Untersuchungsanordnung von Stude insbesondere darum interessant, weil aus den Gesprächen heraus vorwiegend induktiv ermittelt wird, worauf die Kinder beim Sprechen über Sprache Bezug nehmen. Stude führt den Begriff Referenzbereich von Sprachthematisierungen ein, um uneindeutige Begrifflichkeiten und Unklarheiten, auf welchen Aspekt von Sprache die zu untersuchende metasprachliche Äusserung referiert (Stude 2012: 46), zu vermeiden. Diese Referenzbereiche können jeweils etwa metasyntaktische, metasemantische oder metapragmatische Aspekte gleichermassen umfassen. Der Begriff wird für die vorliegende Untersuchung übernommen. Studes Vorschlag folgend werden für die qualitative Analyse der Kinderkommentare möglichst induktiv aus den Daten hergeleitete Referenzbereiche metasprachlicher Reflexion definiert. Der Begriff der Referenzbereiche erlaubt es, von den Kindern 
thematisierte Bereiche unterschiedlicher Natur einander gegenüberzustellen und sie alle als Referenzbereiche zu bezeichnen: Die häufig thematisierte Grossschreibung nach dem Artikel stellt beispielsweise einen detailliert konturierten sprachformalen Referenzbereich dar, während das Referieren auf das ,bessere und hochdeutschere Tönen' einen vorerst unspezifischeren Bereich meint, der - wenngleich über das Schreiben gesprochen wird - auf metaphonologische und metakommunikative Aspekte abzielt. Wie Andresens QuantEinheiten sollen aus den Gesprächen Grössen herausgearbeitet werden, auf die sich die Kinder metasprachlich beziehen. Das müssen keineswegs linguistisch definierte Kategorien sein. Weil es sich oftmals sogar nur bedingt oder gar nicht um sprachliche Grössen handelt, eignet sich Andresens Quant-Terminologie nicht respektive nur sehr bedingt. Als Vorteil in Bezug auf die begriffliche Präzision bringen es die Referenzbereiche zudem mit sich, dass das Referieren auf etwas terminologisch ins Zentrum rückt und unklare, weil sich auf kognitive Grössen beziehende Begriffe wie Wissensbereiche, vermieden werden können.

\subsection{Sprachhandeln und Sprachwissen als Kontinuum}

Die vorangehenden Ausführungen liessen deutlich werden, dass bestehende Forschungen zur Analyse metasprachlicher Kompetenz Unterschiedliches in den Blick nehmen. Sie verbindet keine einheitliche Auffassung darüber, was Metasprache bezeichnet respektive wodurch sich eine metasprachliche Äusserung oder eine metasprachliche Reflexion als solche qualifiziert. Diese Unterschiedlichkeit hat ihre Gründe einerseits in unterschiedlichen methodischen und konzeptionellen Zugängen verschiedener Disziplinen und andererseits im Gegenstand selber: Was vorrangig psycholinguistisch real und relevant ist und konkrete (objekt-)sprachliche Realisierungen überlagert, ist inhaltlich weniger gut beschreibbar. Ein zentrales Wesensmerkmal von Konzeptualisierungen zur Metasprache aber liegt schlicht darin, dass eine sogenannte Objektsprache zu ihr zwar einen Gegenpol bilden mag, sich aber von ihr nicht trennscharf abgrenzen lässt. Vielmehr ist zwischen diesen prototypischen Ausprägungen objekt- und metasprachlicher Äusserungen von einem Kontinuum auszugehen. Dieser Aspekt wird in Abbildung 5.2 veranschaulicht.

Die Abbildung führt zusammenfassend die in Kapitel 5 aufgegriffenen Modelle und Termini auf und ordnet sie auf dem Kontinuum zwischen sogenannter prototypischer Objektsprache und sogenannter prototypischer Metasprache an. Die Übersicht vereint Termini und Modelle mit ganz unterschiedlicher theoretischer Reichweite: Während die Definitionen Weingartens für eine einzelne Studie operationalisiert wurden, ist die Unterscheidung in explizites und impli- 


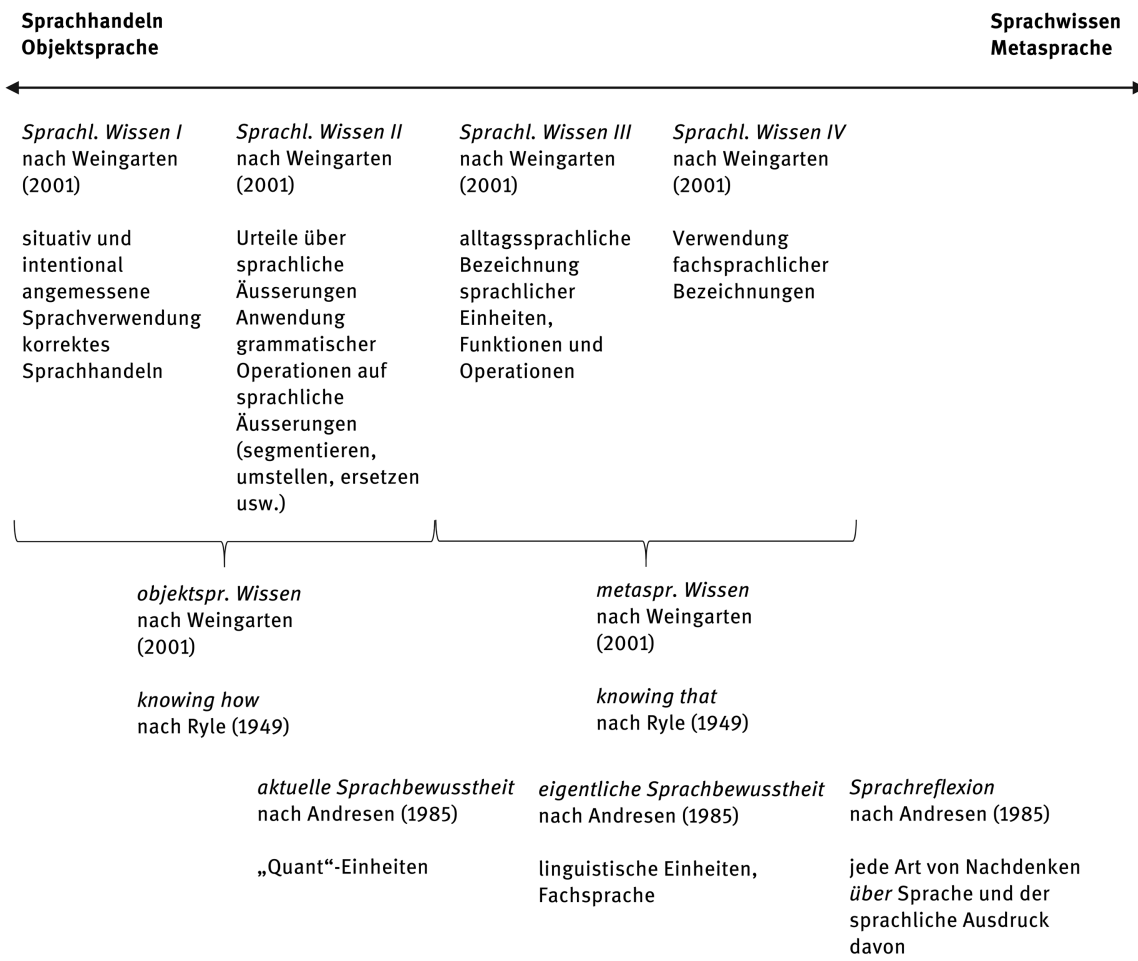

phonological

awareness

(bezieht sich auf die

lautl. Dimension)

Abb. 5.2: Übersicht über Begrifflichkeiten zu Objekt- und Metasprache.

zites Wissen, die auf Polanyi zurückgeht, in der Forschung breit rezipiert worden. Zum Ausdruck gebracht werden sollen die inhaltlichen Unterschiede in der Konzeption von Objekt- und Metasprache oder von Sprachhandeln und Sprachwissen. Die Begriffspaare werden leicht als dichotome Grössen interpretiert, sind es aber im realen Sprachgebrauch nicht. Es gibt zweifelsfrei prototypische Vertreter der Kategorien Objektsprache und Metasprache oder der Kategorien Sprachkönnen und Sprachwissen, dazwischen aber lassen sich sehr viele, in natürlichen Gesprächssituationen vorkommende Äusserungen nur schwer zuordnen, da sie Sprachkönnen eines Individuums ebenso zum Aus- 
druck bringen, wie sie darauf hinweisen, dass dieses über die Fähigkeit verfügt, eine sprachliche Äusserung zu reflektieren.

Die Abbildung illustriert, dass die in den hier erwähnten Studien verwendeten Termini in ihrer Verwendung kaum in Deckung zu bringen sind: Was in einer Studie als metasprachliches Wissen qualifiziert wird, gälte im Kontext einer anderen Untersuchung als Sprachhandeln. Erkenntnisse einzelner Studien miteinander zu vergleichen, wird dadurch nahezu unmöglich. Diese mannigfache und inhaltlich unterschiedliche Verwendung der Termini ist darauf zurückzuführen, dass Sprachhandeln und Sprachwissen / Objektsprache und Metasprache keine klar abgrenzbaren Kategorien darstellen. Die Ausführungen zum Phasen-Modell von Karmiloff-Smith (cop. 1992) (vgl. Kap. 5.3.3) haben genau das in Bezug auf das explizite und implizite Sprachwissen gezeigt: Da zwischen implizitem und explizitem Wissen Umstrukturierungsprozesse vonstattengehen, vermischen sich die beiden Wissenstypen automatisch. Daher wird es den Lern- und Entwicklungsmechanismen nicht gerecht, zwei dichotome Kategorien anzunehmen. Der empirische Weg zu einer Differenzierung dieser Kategorien und Termini ist besonders darum vielversprechend, weil er $\mathrm{zu}$ Kenntnissen über das sprachliche Wissen von Kindern führen könnte, an die danach sprachdidaktische und diagnostische Überlegungen anknüpfen könnten. Derzeit sind empirisch abgesicherte Erkenntnisse über die Bedeutung metasprachlicher Fähigkeiten für Sprachaneignungsprozesse nur beschränkt vorhanden. Die empirische Aufarbeitung metasprachlicher Kompetenzen von Kindern in der Forschung stellt derzeit nach wie vor ein Desiderat dar. Das gilt insbesondere für Herangehensweisen, wie sie für die hier präsentierte Studie gewählt wird. Diese erhebt und beschreibt metasprachliches Wissen der Kinder ausgehend von deren Schreibhandlung (vgl. zum Vorgehen Kap. 9.1.6) und setzt damit bei dem Erfahrungsstand der einzelnen Kinder an. Dahinter steckt das Ziel, über die empirische Auseinandersetzung mit Funktionen metasprachlichen Wissens, das die Kinder basierend auf ihren eigenen Schreibhandlungen rekonstruieren, das Verhältnis zwischen verschiedenen Sprachwissenstypen zueinander zu erhellen. Die Voraussetzung dafür ist eine Klärung der Begrifflichkeiten. Für die Konzeptualisierung der empirischen Untersuchung wird darum an späterer Stelle (vgl. Kap. 7.1) eine terminologische Differenzierung vorgenommen, die die Termini Metasprache und Sprachwissen durch die Begriffe implizites Wissen, explizites Wissen und expliziertes Wissen ersetzt. 


\subsection{Zusammenfassung Kapitel 5}

„Sprache ist für mich eine reihe von lauten

mit einander verbunden, um Wörter, Sätze zu bilden.“

Datenkorpus $^{35}$, Schüler $6 \mathrm{ml} 1$

Mit dem Eintritt der Kinder in die Schriftlichkeit eröffnet sich ihnen das Potenzial, über Sprache zu sprechen und zu verhandeln, in einer neuen Dimension. Sie nehmen Formen des metasprachlichen Reflektierens und Sprechens aus der Mündlichkeit zwar bereits mit, werden durch die geschriebene Sprache aber in der metasprachlichen Auseinandersetzung neu stimuliert. Formen der Sprachbetrachtung begleiten die Schriftaneignung von den basalen Grundfertigkeiten an bis hin zur Entfaltung literaler Textkompetenz. Sie richtet sich potenziell auf unterschiedliche Aspekte von Sprache: auf ihre pragmatische und kommunikative Funktion etwa, auf ihre typologische Ausprägung oder auf grammatische Kategorien und Modelle, die als ihr zugrundeliegend angenommen werden. Aus dem Lautstrom, den das Sprechen auszeichnet, werden in der schriftlichen Varietät neue Kategorien, wie es der oben zitierte Junge beschreibt: Aus der Reihe von Lauten werden Wörter und Sätze, die miteinander verbunden sind. Tätigkeiten der Sprachbetrachtung begleiten das Schreiben als eine Art kognitiver Begleit- und Kontrollhandlungen und gehen aus der Sprachproduktion wiederum hervor. Insbesondere für den schulischen Kontext bedarf es einer Klärung dafür, mit welchen Termini und Kategorien über Sprache gesprochen wird und welche sprachlichen Elemente oder Konstruktionen der Sprachbetrachtung und -reflexion zugeführt werden. Hier haben die vorangehenden Ausführungen verschiedene Ansätze einander gegenübergestellt und ihre jeweiligen Vorzüge herausgehoben. Als Beispiel für ein Konzept, das der Forschung eine konkrete Begrifflichkeit an die Hand gibt, ist die Studie von Andresen (1985). Sie bringt im Anschluss an Leontev (1975) Quant-Einheiten in die Diskussion ein. Dabei handelt es sich um pragmatisch relevante, aber nicht zwingend durch linguistische Operationen ermittelbare Grössen, die sich zwar der Terminologie der traditionellen Grammatik (Wort, Satz etc.) bedienen, die interessierenden sprachlichen Strukturen aber durch das Präfix als psycholinguistische Kategorien auszeichnen. Andresen schlägt damit ein Konzept vor, wie metasprachliche Äusserungen bezeichnet werden könnten. Gleichzeitig nimmt sie eine Differenzierung in verschiedene Stufen metasprachlicher Be-

35 Der Ausschnitt stammt aus einem Schülerfragebogen. Der Junge äusserte sich dazu, was Sprache für ihn bedeute. Die offene Frage im Fragebogen wurde nicht für Auswertungen im Rahmen der vorliegenden Studie erhoben. 
wusstheit vor. Dieser Aspekt wird umfassend von Karmiloff-Smith (cop. 1992) bearbeitet. Die Ausdifferenzierung verschiedener Grade sprachlicher Bewusstheit und sprachlichen Wissens zeichnet ihre Modellierung aus. KarmiloffSmith (cop. 1992) bezieht sich terminologisch auf die Unterscheidung zwischen implizitem und explizitem Wissen, nimmt aber eine Feingliederung in verschiedene Grade impliziten und expliziten Wissens vor. Das Verdienst ihres konnektionistisch-konstruktivistischen Lernmodells ist die Erklärung dafür, wie Sprachwissen über verschiedene Stufen von implizitem zu explizitem Wissen wird. Zudem stellt das Modell ein wichtiges Charakteristikum von Lernprozessen heraus: Die kognitiven Umstrukturierungsprozesse führen laut Karmiloff-Smith (cop. 1992) in der sprachlichen Performanz immer wieder zu Fehlern, Hyperkorrekturen oder anderen Auffälligkeiten. Diese sind vor dem Hintergrund dieser Restrukturierung auf der Sprachwissensebene aber Ausdruck durchaus positiver Verarbeitungsprozesse. Auf diese Modellierung geht die Bezeichnung der sogenannten U-Kurven-Dynamik zurück, die zum Ausdruck bringt, dass sich diese Restrukturierungsprozesse auf der sprachlichen Oberfläche als - nur scheinbarer - Rückschritt im Lernprozess zeigen.

Die verschiedenen Termini, deren sich theoretische Modellierungen metasprachlicher Zugriffe auf Wissensstrukturen bedienen, geben insgesamt ein diffuses Bild dessen ab, was unter Metasprache, Sprachbewusstsein oder implizitem und explizitem Wissen gemeint ist. Der Grund für die terminologische Uneinheitlichkeit liegt einerseits in den unterschiedlichen Forschungsinteressen und -methoden der am Gegenstand interessierten Disziplinen. Andererseits ist sie darauf zurückzuführen, dass die Thematik stark von theoretischen Konzepten dominiert wird und empirische Untersuchen für den deutschsprachigen Raum sehr rar sind. Das betrifft insbesondere den Zusammenhang zwischen Schriftsprach- und Textaneignung und metasprachlichen Kompetenzen. Abbildung 5.3 illustriert zudem einen weiteren wichtigen Aspekt, den die vorangehenden Ausführungen herausgearbeitet haben: Die Konzepte Metasprache, Sprachwissen oder Sprachbewusstsein bezeichnen sowohl kognitive Qualitäten des Individuums als auch Konstrukte der Sprachwissenschaft, die diese entweder theoretisch oder empirisch hergeleitet hat. Diese Termini beziehen sich teilweise auf beobachtbare Aspekte des Sprachgebrauchs (wie sprachlichen Strukturen in Kindertexten), teilweise aber auch auf kognitive Voraussetzungen, die nicht direkt beobachtbar sind. Auf sie wird basierend auf Sprachhandlungen des Kindes zurückgeschlossen. Als Sprachhandlung kommt wiederum Verschiedenes in Frage: Es kann sich dabei ebenfalls um Kindertexte handeln oder etwa um mündliche Äusserungen der Kinder über einen sprachlichen Aspekt.

Eine empirische Herangehensweise verlangt eine Klärung nicht nur in Bezug darauf, mit welchen Termini der Gegenstand zu bezeichnen ist, der ver- 


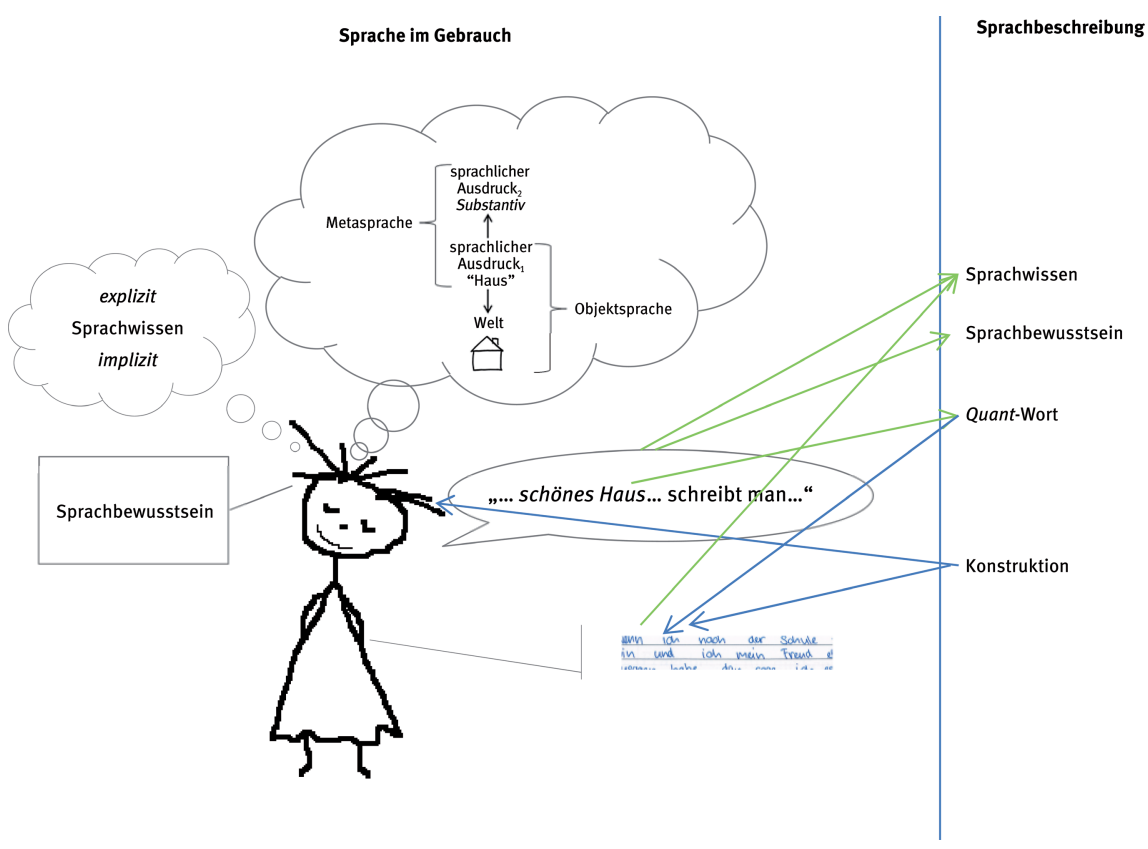

Abb. 5.3: Metasprache im Sprachgebrauch und in der Sprachbeschreibung. Anm.: Die blauen Pfeile lesen sich als bezeichnen xy als, die grünen als führt zum Terminus / zum Konzept. Quellen: Abbildung in Gedankenblase: Bredel 2007: 26 (vgl. Abb. 5.1), Kindertext: Ausschnitt aus dem Datenkorpus der vorliegenden Studie (Fragebogen 4ml13).

sprachlicht wird - Metasprache, Sprachwissen -, sondern insbesondere auch, welche Handlungen ein Individuum vollzieht, wenn es sich metasprachlich äussert. Der Begriff der Sprachbewusstheit drückt diese Bindung an das Individuum aus, da sie diesem eine kognitive Kompetenz zuschreibt. In präzisere Bestimmungsmerkmale überführt Bredel (2007) diese Handlungen, indem sie beschreibt, wodurch sich metasprachliche Diskurse als solche qualifizieren. Sie nennt dafür die folgenden Merkmale: die Distanzierung von der beobachteten sprachlichen Struktur, die Deautomatisierung des Sprachhandlungsprozesses und die Dekontextualisierung des betrachteten Phänomens. Vor dem Hintergrund der Daten, die der vorliegenden Studie zugrunde liegen, werden diese drei Merkmale um einen vierten Bereich respektive um eine Präzisierung des letztgenannten ergänzt. Neben der Dekontextualisierung eines Phänomens aus dem sprachlichen Kontext wird die Re-Kontextualisierung in den Text- und Sprachproduktionskontext als wichtig angenommen.

Es ist ebenfalls der empirischen Herangehensweise geschuldet, dass die Frage, welche sprachlichen Strukturen von den Kindern metasprachlich the- 
matisiert werden, breiter diskutiert und offener angegangen werden muss, als es die vorgestellten Ansätze erlauben. Gerade wenn metasprachliche Äusserungen ganz verschiedener Art, also bezogen auf unterschiedliche und unterschiedlich stark umrissene sprachliche Phänomene, erfasst werden sollen, genügen weder die Termini und Kategorien der Schulgrammatik noch deren Ergänzung um das von Andresen (1985) vorgeschlagene Präfix zur Markierung der psycholinguistischen Relevanz der Struktur (beispielsweise Quant-Wort). Um der Analyse in der vorliegenden Studie einen geeigneten Terminus zugrunde zu legen, der verschieden konturierte und auf verschiedenen (sprachlichen) Ebenen angesiedelte Bereiche zu fassen vermag, wird in Anlehnung an Stude von Referenzbereichen metasprachlicher Reflexion gesprochen. Diese Referenzbereiche können jeweils metasyntaktische, metasemantische oder metapragmatische Aspekte gleichermassen umfassen. Damit rückt terminologisch ins Zentrum, was die vorliegende Studie erhellen möchte: Die Frage, worauf die Kinder referieren, wenn sie sich über Sprach- und Schreibprozesse äussern. Es interessiert also nicht so sehr, in welcher Form respektive anhand welcher Termini die Kinder das tun, sondern es wird betrachtet, was ihnen an sprachlichen oder textuellen Strukturen auffällt und wie sie Schreibhandlungen begründen. 